\title{
The simultaneous equations models for the application in forestry
}

\author{
Fuxiang Liu \\ Department of statistics at School of economics, Harbin University of Commerce, Heilongjiang Province, \\ PR China \\ liufuxiang2009@163.com
}

Keywords: compatible biomass equations, simultaneous equations, measure error model

\begin{abstract}
In accordance with weakness of traditional biomass models in which the sum of the above- and below-ground tree components was not equal to the whole tree, compatible biomass equations were developed for the above- and below-ground tree components of 11 kinds of tree species in Heilongjiang Province. The data used to develop biomass models are from 299 trees that were collected from 69 sample plots, and represented a wide range of stand and site conditions in Heilongjiang Province. Based on the total biomass model as restrictions, the compatible tree biomass equations for each component (stems, branches, foliages, and roots) were separately established by considering diameter at breast height (D) as independent variables in the form of simultaneous equations with measure error of independent variables. The evaluation and validation procedures for tree biomass models were performed by using the following statistical criterions: the coefficient of determination (R2), sum square of error (SEE), Mean Bias (MB), and Mean Absolute Bias (MAB). The results showed that although parameter estimation accuracy of compatible biomass equations was not better than traditional biomass models, the compatible biomass equations could effectively overcome the weakness of un-compatible of traditional biomass models.
\end{abstract}

\section{Introduction}

Forest biomass estimation is the groundwork of analyzing carbon cycle and its dynamics in terrestrial ecosystems. So there is a current need for reliable biomass models for total tree and individual tree components. First of all, which have to produce reliable biomass estimates applicable over the wide range; secondly, which should be based on the variables that are normally measured in forest inventories, or which can be estimated easily and reliably from inventory data; thirdly, one desirable feature of the tree components equations is biomass compatible, i.e. that the sum of the predictions for the tree component equals the prediction for the whole tree (Kozak 1974, Cunia and Briggs 1984, Parresol 1999, 2001). The estimation models for tree biomass had the problem of un-compatibility between total forest biomass and its components(stem wood, branches, foliages and roots) as follows Table (1), where for traditional model $w=a D^{b}$ as examples with Korean pine plantation, Mono maple and Manchurian ash, the total biomass is not equal to the sum of their components; even the aboveground biomass is equal to the sum of biomass of stem, branches and foliages. There are other two problems in current biomass models:(i) Forest biomass estimates mainly consider aboveground component(stem 、 branch 、 foliage), and underground biomass are estimated less (Williams, et al., 2005,Bond-Lamberty et al., 2002). Due to the difficult to determine below-ground biomass, the sample size is rarely, and consequently the accuracy of fit and predictor of the models with this small number of samples is unsatisfactory. (ii) Most of current researches are more concentrated on the plantation, furthermore tree species are relatively few.

Based on method of the simultaneous equations and measure error model, the compatible tree biomass models were developed for above- and below-ground tree components for 11 kinds of main species in Heilongjiang Province. 
Table 1 An example of incompatibility of traditional models with equation (1)

\begin{tabular}{ccccccc}
\hline Tree species & $\mathrm{D}(\mathrm{cm})$ & $\mathrm{H}(\mathrm{cm})$ & $\triangle 1(\mathrm{~kg})$ & $\triangle 1(\%)$ & $\triangle 2(\%)$ & $\triangle 2(\mathrm{~kg})$ \\
& 33 & 12.3 & 110.603 & 21.38618 & -37 & -111.97 \\
KP plantation & 25.6 & 11.9 & -12.785 & -5.8998 & -5.2 & -75.72 \\
& 18.5 & 9.9 & 19.855 & 15.10898 & -60 & -33.65 \\
& 19.5 & 12.5 & -9.186 & -7.91405 & -19.76 & -56.92 \\
Mono maple & 32.5 & 15.3 & -60.468 & -18.8779 & -45.67 & -99.86 \\
& 23 & 13.4 & 13.320 & 67.23944 & -37.25 & -72.46 \\
Manchurian & 16.1 & 12.8 & 30.948 & 36.06704 & -58.65 & -23.49 \\
ash & 14.1 & 15.2 & -63.713 & -6.87664 & -11 & -59.942 \\
& 8.2 & 16.1 & 50.298 & 29.53442 & -46 & -49.57 \\
& 16.7 & 14.1 & -3.083 & -4.63676 & -12 & -10.45 \\
\hline
\end{tabular}

Note: $\triangle 1=$ total-(stem+branch+foliage+root), $\triangle 1 \%=\triangle 1 /$ total $* 100$;

$\triangle 2=$ total of aboveground-( stem+branch + foliage), $\triangle 2 \%=\triangle 2$ / total of aboveground $* 100$

\section{Materials and Method}

The data were collected from 16 counties in Heilongjiang Province of Northeast China, ranging across about $1120 \mathrm{~km}$ in NS and $930 \mathrm{~km}$ in WE $\left(43^{\circ} 25^{\prime}-53^{\circ} 33^{\prime} \mathrm{N}, 121^{\circ} 11^{\prime}-135^{\circ} 05^{\prime} \mathrm{E}\right)$ and the total land area is $454,000 \mathrm{~km} 2$.

\subsection{Study areas}

The climate features is continental monsoon climate with cold winters and warm summers. The average annual temperature is $4^{\circ} \mathrm{C} \sim 4^{\circ} \mathrm{C}$, the average annual rainfall is $450 \mathrm{~mm}$ to $700 \mathrm{~mm}$, and Frost-free period is between $100 \mathrm{~d}$ to $160 \mathrm{~d}$. Main species are Korean Pine (Pinus koraiensis), Mongolian pine (Pinus sylvestris var. mongolica), Korean spruce (Picea koraiensis), Jezo spruce(Picea jezoensis), Amur fir (Abies nephrolepis), Dahurian Larch (Larix gmelini), Changbai Larch ( Larix olgensis), Manchurian ash (Fraxinus mandshurica), Walnut (Junglus mandshurica), Cork tree (Phellodendron amurense), Basswood (Tilia amurensis, T. mandshurica), Mono maple (Acer mono), elm (Ulmus propinqua, Ulmus laciniata), Mongolian oak (Quercus mongolica), Costata birch (Betula costata), White birch (Betula platyphlla), Dahurian birch (Betula davuria), papen (Populus davidiana, Populus ussuriensis), and etc.

\subsection{Data}

The whole data set (297 trees from 64 sample plots) was randomly divided into two data sets: fitting data set (254 samples) and validation data set (43 samples).

Table 2. Summary of sample trees

\begin{tabular}{|c|c|c|c|c|c|c|c|}
\hline \multirow[b]{2}{*}{ species } & \multirow[b]{2}{*}{ Attribute } & \multicolumn{3}{|c|}{ Modeling data } & \multicolumn{3}{|c|}{ Validation data } \\
\hline & & Minimum & $\begin{array}{l}\text { Mean } \\
\text { (s.d.) }\end{array}$ & Maximum & Minimum & $\begin{array}{l}\text { Mean } \\
\text { (s.d.) }\end{array}$ & Maximum \\
\hline \multirow{2}{*}{$\begin{array}{c}1 \\
\mathrm{~N}=26 \\
\mathrm{~N}^{+}=4\end{array}$} & $\mathrm{D}(\mathrm{cm})$ & 4.8 & $\begin{array}{c}17.39 \\
(1.30)\end{array}$ & 30.8 & 19 & $\begin{array}{l}24.15 \\
(3.34)\end{array}$ & 33 \\
\hline & $\mathrm{H}(\mathrm{m})$ & 4.68 & $\begin{array}{c}11.54 \\
(0.58)\end{array}$ & 16.2 & 9.9 & $\begin{array}{c}11.65 \\
(0.59)\end{array}$ & 12.5 \\
\hline \multirow{2}{*}{$\begin{array}{c}2 \\
\mathrm{~N}=16 \\
\mathrm{~N}^{+}=2\end{array}$} & $\mathrm{D}(\mathrm{cm})$ & 12.2 & $\begin{array}{l}23.2 \\
(2.0)\end{array}$ & 38.7 & 17.4 & $\begin{array}{c}19 \\
(1.6)\end{array}$ & 20.6 \\
\hline & $\mathrm{H}(\mathrm{m})$ & 15.1 & $\begin{array}{l}44.09 \\
(0.31)\end{array}$ & 19.7 & 16 & $\begin{array}{l}16.6 \\
(0.6)\end{array}$ & 17 \\
\hline \multirow{2}{*}{$\begin{array}{r}3 \\
N=18 ; \\
N^{+}=5\end{array}$} & $\mathrm{D}(\mathrm{cm})$ & 2.30 & $\begin{array}{c}3.37 \\
(0.86)\end{array}$ & 5.60 & 15.5 & $\begin{array}{c}20.43 \\
(4.15)\end{array}$ & 28.7 \\
\hline & $\mathrm{H}(\mathrm{m})$ & 8 & $\begin{array}{l}11.8 \\
(2.2)\end{array}$ & 15 & 12.5 & $\begin{array}{c}16.53 \\
(3.54)\end{array}$ & 23.6 \\
\hline $\begin{array}{c}4 \\
N=27 ;\end{array}$ & $\mathrm{D}(\mathrm{cm})$ & 4.8 & $\begin{array}{r}15.3 \\
(1.12) \\
\end{array}$ & 27.0 & 16.1 & $\begin{array}{l}23.86 \\
(4.75)\end{array}$ & 32.5 \\
\hline
\end{tabular}




\begin{tabular}{|c|c|c|c|c|c|c|c|}
\hline $\mathrm{N}^{+}=3$ & $\mathrm{H}(\mathrm{m})$ & 6.2 & $\begin{array}{c}1.21 \\
(0.50)\end{array}$ & $\begin{array}{l}18.6 \\
5\end{array}$ & 12.8 & $\begin{array}{l}13.83 \\
(0.75)\end{array}$ & 15.3 \\
\hline 5 & $\mathrm{D}(\mathrm{cm})$ & 6.0 & $\begin{array}{l}20.2 \\
(1.86)\end{array}$ & 33.4 & 8.2 & $\begin{array}{l}14.45 \\
(2.29)\end{array}$ & 18.8 \\
\hline $\begin{array}{c}\mathrm{N}=18 \\
\mathrm{~N}^{+}=4\end{array}$ & $\mathrm{H}(\mathrm{m})$ & 9.20 & $\begin{array}{l}16.97 \\
(0.98)\end{array}$ & $\begin{array}{l}22.5 \\
0\end{array}$ & 8.9 & $\begin{array}{l}13.57 \\
(1.61)\end{array}$ & 16.1 \\
\hline $\begin{array}{c}6 \\
N=16 .\end{array}$ & $\mathrm{D}(\mathrm{cm})$ & 8.1 & $\begin{array}{c}19.5 \\
(1.66)\end{array}$ & 28.8 & 14.7 & $\begin{array}{l}16.15 \\
(1.45)\end{array}$ & 17.6 \\
\hline $\begin{array}{l}\mathrm{N}=16 \\
\mathrm{~N}^{+}=2\end{array}$ & $\mathrm{H}(\mathrm{m})$ & 9.6 & $\begin{array}{l}17.94 \\
(0.96)\end{array}$ & 23.1 & 12.7 & $\begin{array}{l}15.25 \\
(2.55)\end{array}$ & 17.8 \\
\hline $\begin{array}{c}7 \\
\mathrm{~N}=27\end{array}$ & $\mathrm{D}(\mathrm{cm})$ & 4.2 & $\begin{array}{l}16.45 \\
(1.68)\end{array}$ & 37.1 & 7.2 & $\begin{array}{c}24.5 \\
(8.65)\end{array}$ & 33.2 \\
\hline $\mathrm{N}^{+}=3$ & $\mathrm{H}(\mathrm{m})$ & 5 & $\begin{array}{l}11.5 \\
(0.74)\end{array}$ & 18.7 & 6.3 & $\begin{array}{l}13.76 \\
(3.73)\end{array}$ & 17.8 \\
\hline $\begin{array}{c}8 \\
N=20 .\end{array}$ & $\mathrm{D}(\mathrm{cm})$ & 10.0 & $\begin{array}{l}18.7 \\
(1.31)\end{array}$ & 28.7 & 12.1 & $\begin{array}{c}20.6 \\
(5.88)\end{array}$ & 31.9 \\
\hline $\begin{array}{l}\mathrm{N}=2 \mathrm{O} \\
\mathrm{N}^{+}=3\end{array}$ & $\mathrm{H}(\mathrm{m})$ & 10.9 & $\begin{array}{l}15.8 \\
(0.75)\end{array}$ & 21.4 & 11.2 & $\begin{array}{l}15.73 \\
(2.57)\end{array}$ & 20.1 \\
\hline $\begin{array}{c}9 \\
N=18 .\end{array}$ & $\mathrm{D}(\mathrm{cm})$ & 10.4 & $\begin{array}{c}19.3 \\
(1.28)\end{array}$ & 31.3 & 11 & $\begin{array}{c}14.47 \\
(4.50)\end{array}$ & 26.8 \\
\hline $\begin{array}{l}\mathrm{N}=18 \\
\mathrm{~N}^{+}=4\end{array}$ & $\mathrm{H}(\mathrm{m})$ & 9.80 & $\begin{array}{l}16.22 \\
(0.82)\end{array}$ & $\begin{array}{l}22.7 \\
0\end{array}$ & 10 & $\begin{array}{c}13.4 \\
(2.76)\end{array}$ & 19.9 \\
\hline $\begin{array}{c}10 \\
\mathrm{~N}=29\end{array}$. & $\mathrm{D}(\mathrm{cm})$ & 7.3 & $\begin{array}{c}18.9 \\
(1.65)\end{array}$ & 47.0 & 19 & $\begin{array}{l}24.15 \\
(3.34)\end{array}$ & 33 \\
\hline $\begin{aligned} \mathrm{N} & =29 \\
\mathrm{~N}^{+} & =4\end{aligned}$ & $\mathrm{H}(\mathrm{m})$ & 8.2 & $\begin{array}{l}13.84 \\
(0.65)\end{array}$ & 19.7 & 9.9 & $\begin{array}{l}11.65 \\
(0.59)\end{array}$ & 12.5 \\
\hline $\begin{array}{c}11 \\
N=39\end{array}$ & $\mathrm{D}(\mathrm{cm})$ & 3.6 & $\begin{array}{l}15.42 \\
(1.21)\end{array}$ & 35.1 & 3 & $\begin{array}{c}12.89 \\
(0.37)\end{array}$ & 24.8 \\
\hline $\mathrm{N}^{+}=9$ & $\mathrm{H}(\mathrm{m})$ & 3.6 & $\begin{array}{l}13.09 \\
(1.10)\end{array}$ & 46.8 & 4.8 & $\begin{array}{l}11.73 \\
(2.58)\end{array}$ & 36 \\
\hline $\begin{array}{c}\text { Total } \\
\mathrm{N}=254 .\end{array}$ & $\mathrm{D}(\mathrm{cm})$ & 3.6 & $\begin{array}{l}17.961 \\
(0.47)\end{array}$ & 47.0 & 3 & $\begin{array}{c}18.24 \\
(1.28)\end{array}$ & 33 \\
\hline $\begin{array}{l}\mathrm{N}=254 ; \\
\mathrm{N}^{+}=43\end{array}$ & $\mathrm{H}(\mathrm{m})$ & 3.6 & $\begin{array}{l}14.37 \\
(0.35)\end{array}$ & 74.0 & 5 & $\begin{array}{c}13.7 \\
(0.61)\end{array}$ & 24 \\
\hline
\end{tabular}

Note: $\mathrm{D}$ and $\mathrm{H}$ stand separately for diameter at breast height and total tree height. S.d. is Std. Error of Mean. $\mathrm{N}^{+}$is the number of Validation data. Tree species: 1- Korean pine plantation; 2- Mongolian pine plantation; 3-Spruce; 4- Mono maple; 5- Manchurian ash; 6-aspen; 7-Mongolian oak; 8-Larch; 9-Fir; 10- Korean pine; 11- Dahurian birch

\section{Compatible Tree Biomass Model}

AffiliationThe traditional tree biomass equations were fitted separately for each component and total biomass by using of Equation (1). As a result, they were not compatible i.e. the sum of predicted for all components was not equal to the prediction for total tree biomass.

$$
w=a D^{b} ;
$$

Where $w$ trees for biomass, a b and c are corresponding parameters.

Based on the total biomass model as restrictions,the compatible tree biomass equations for each component (stem, branches, foliages, and roots) were separately developed by using the theory of simultaneous equations and measure error mode with Equation(1).

In case of Equation (1), compatible biomass model was constructed as follows:

$$
\left\{\begin{array}{c}
w_{s}=a_{1}(x) D^{b_{1}} \\
w_{b}=a_{2}(x) D^{b_{2}} \\
w_{l}=a_{3}(x) D^{b_{3}} \\
w_{r}=a_{4}(x) D^{b_{4}} \\
w_{t}=c_{0} D^{b_{0}}=a_{1}(x) D^{b_{1}}+a_{2}(x) D^{b_{2}}+a_{3}(x) D^{b_{3}}+a_{4}(x) D^{b_{4}}
\end{array}\right.
$$

Where $w_{s}, w_{b}, w_{l}, w_{r}$ and ${ }^{w_{t}}$ are biomass of stem, branch, foliage, root, and the total tree,separately; 
$a_{1}(D), b_{1}, a_{2}(D), b_{2}, a_{3}(D), b_{3}, a_{4}(D), b_{4}, c_{0}$ and $b_{0}$ are parameters to be estimated.

In Equation(3), $a_{1}(D), a_{2}(D), a_{3}(D), a_{4}(D)$ should be introduced as follows:

$$
\begin{aligned}
& a_{1}(D)=c_{0} c_{1} D^{b 0} /\left(c_{1} D^{b 1}+c_{2} D^{b 2}+c_{3} D^{b_{3}}+c_{4} D^{b_{4}}\right), a_{2}(D)=c_{0} c_{2} D^{b 0} /\left(c_{1} D^{b 1}+c_{2} D^{b 2}+c_{3} D^{b_{3}}+c_{4} D^{b_{4}}\right) \\
& a_{3}(D)=c_{0} c_{3} D^{b 0} /\left(c_{1} D^{b 1}+c_{2} D^{b 2}+c_{3} D^{b_{3}}+c_{4} D^{b_{4}}\right), a_{4}(D)=c_{0} c_{4} D^{b 0} /\left(c_{1} D^{b 1}+c_{2} D^{b 2}+c_{3} D^{b_{3}}+c_{4} D^{b_{4}}\right) \\
& \text { Let } r_{1}=c_{2} / c_{1}, r_{2}=c_{3} / c_{1} ; \quad r_{3}=c_{4} / c_{1} ; k_{1}=b_{2}-b_{1} ; k_{2}=b_{3}-b_{1} ; k_{3}=b_{4}-b_{1}
\end{aligned}
$$

Then Equation (2) is transformed into as follows:

$$
\left\{\begin{array}{c}
w_{s}=c_{0} D^{b_{0}} /\left(1+r_{1} D^{k_{1}}+r_{2} D^{k_{2}}+r_{3} D^{k_{3}}\right) \\
w_{b}=c_{0} c_{1} D^{k_{1}+b_{0}} /\left(1+r_{1} D^{k_{1}}+r_{2} D^{k_{2}}+r_{3} D^{k_{3}}\right) \\
w_{l}=c_{0} c_{2} D^{k_{2}+b_{0}} /\left(1+r_{1} D^{k_{1}}+r_{2} D^{k_{2}}+r_{3} D^{k_{3}}\right) \\
w_{r}=c_{0} c_{3} D^{k_{3}+b_{0}} /\left(1+r_{1} D^{k_{1}}+r_{2} D^{k_{2}}+r_{3} D^{k_{3}}\right)
\end{array}\right.
$$

The parameter estimates of nonlinear simultaneous equations with measure error (Equation (3)) were performed using Newton-tang Algorithm implemented in ForStat 2.1 software (CAF, 2009). Goodness-of-fit of the alternative models were assessed on the basis of the statistics of the residual sum of squares, RSS, and the coefficient of determination ( R2).

The validation procedures for traditional biomass models and the compatible biomass models developed in this paper were performed by using the following statistical criterions: the coefficient of determination (R2), sum square of error (SSE), Mean Bias (MB), and Mean Absolute Bias (MAB).

$$
\mathrm{R}^{2}=1-\sum\left(y_{i}-\hat{y}_{i}\right)^{2} / \sum\left(y_{i}-\bar{y}\right)^{2} ; \mathrm{MAB}=\frac{1}{n} \sum_{i=1}^{n}\left|y_{i}-\hat{y}_{i}\right|, \mathrm{MB}=\frac{1}{n} \sum_{i=1}^{n}\left(y_{i}-\hat{y}_{i}\right) ; \mathrm{SSE}=\sum\left(y_{i}-\hat{y}_{i}\right)^{2}
$$

\begin{tabular}{|c|c|c|c|c|c|c|c|c|}
\hline \multirow{2}{*}{ Species } & \multicolumn{8}{|c|}{ Parameters estimated } \\
\hline & $\mathrm{c}_{0}$ & $\mathrm{~b}_{0}$ & $\mathrm{r}_{2}$ & $\mathrm{k}_{2}$ & $r_{3}$ & $\mathrm{k}_{3}$ & $\mathrm{r}_{4}$ & $\mathrm{k}_{4}$ \\
\hline $\begin{array}{c}\text { KP } \\
\text { plantation }\end{array}$ & 24.14086 & 1.03140 & 0.000840 & 0.64832 & 0.21412 & -0.042039 & 0.48287272 & -0.0230 \\
\hline $\begin{array}{c}\text { MP } \\
\text { plantation }\end{array}$ & 25.17351 & 1.00058 & 0.031350 & 0.07854 & 0.03978 & -0.056128 & 0.14073115 & 0.10040 \\
\hline Spruce & 17.38124 & 1.07325 & 0.005069 & 0.42985 & 0.03599 & 0.0487775 & 0.02062650 & 0.34267 \\
\hline $\begin{array}{l}\text { Mono } \\
\text { maple }\end{array}$ & 19.12495 & 1.04872 & 0.002434 & 0.52693 & 0.00263 & 0.3412480 & 0.544307 & -0.052 \\
\hline $\begin{array}{l}\text { Manchurian } \\
\text { ash }\end{array}$ & 36.8943 & 0.95334 & 1.398060 & -0.2455 & 3.336398 & -0.41341 & 11.12461 & -0.41102 \\
\hline Poplar & 46.0008 & 0.94360 & 0.002397 & 0.41533 & 0.024524 & 0.02983 & 0.157821 & 0.09695 \\
\hline $\begin{array}{l}\text { Mongolian } \\
\text { oak }\end{array}$ & 83.6952 & 0.91440 & 6.148357 & -0.3639 & 3.102146 & -0.48562 & 0.25736 & 0.06634 \\
\hline Larch & 84.1796 & 0.88250 & 0.116760 & 0.07156 & 6.116512 & -0.45477 & 0.43551 & -0.02923 \\
\hline Fir & 54.0066 & 0.91293 & 0.131415 & 0.01541 & 0.05126 & 0.100863 & 0.20059 & 0.07070 \\
\hline Korean pine & 66.2385 & 0.90011 & 1.529737 & -0.2375 & 0.04931 & -0.011538 & 0.02810 & 0.24183 \\
\hline $\begin{array}{l}\text { Dahurian } \\
\text { Birch }\end{array}$ & 33.28051 & 1.00933 & 0.017472 & 0.34220 & 0.01654 & 0.150447 & 2.94250 & -0.20181 \\
\hline
\end{tabular}

\section{Results and Discussion}

The following is the results of fitting, estimating compatible biomass models.

Table 3 The Results of compatible biomass models fitting with Equation (1) 
Table 4 the Results of compatible biomass models fitting with Equation (1)

\begin{tabular}{|c|c|c|c|c|c|c|c|c|c|c|}
\hline \multirow{3}{*}{ species } & \multicolumn{10}{|c|}{ statistical criterions } \\
\hline & \multicolumn{4}{|c|}{$\mathrm{R}^{2}$} & \multirow{2}{*}{ MAB } & \multirow{2}{*}{ MB } & \multicolumn{4}{|c|}{ SSE } \\
\hline & WS & WB & WL & WR & & & WS & WB & WL & WR \\
\hline KP plantation & .9871 & .9250 & .7748 & .9463 & 5.95 E-11 & $4.046 \mathrm{E}-16$ & 0.4890 & 0.1116 & 0.1140 & 0.1027 \\
\hline MP plantation & .8120 & .6781 & .8088 & .8169 & $1.357 \mathrm{E}-10$ & $3.155 \mathrm{E}-11$ & 5.4137 & 1.1056 & 0.1214 & 0.8233 \\
\hline Spruce & .9923 & .5997 & .7060 & .8981 & 3.037E-10 & 3.078E-11 & 0.1459 & 0.0394 & 0.0034 & 0.2728 \\
\hline Mono maple & .9966 & .9698 & .9594 & .9806 & $5.001 \mathrm{E}-11$ & $6.188 \mathrm{E}-12$ & 0.0252 & 0.0194 & 0.0099 & 0.0392 \\
\hline Manchurian ash & .9918 & .9350 & .8052 & .9130 & 4.119E-11 & $1.002 \mathrm{E}-11$ & 0.1504 & 0.1113 & 0.0143 & 0.1878 \\
\hline Poplar & .8489 & .6930 & .7180 & .7415 & $3.436 \mathrm{E}-11$ & $1.447 \mathrm{E}-11$ & 1.3944 & 0.1961 & 0.0447 & 0.4681 \\
\hline Mongolian oak & .9249 & .9774 & .772 & .9422 & 8.328E-11 & $1.306 \mathrm{E}-11$ & 0.2710 & 0.8691 & 0.0323 & 1.8315 \\
\hline Larch & .9259 & .8685 & .3467 & .9266 & $1.926 \mathrm{E}-09$ & $2.934 \mathrm{E}-12$ & 1.9696 & 0.0150 & 0.0513 & 0.1033 \\
\hline Fir & .8933 & .6868 & .8782 & .8317 & $1.515 \mathrm{E}-10$ & $2.551 \mathrm{E}-11$ & 3.1645 & 0.3131 & 0.3372 & 0.3409 \\
\hline Korean pine & .9723 & .6930 & .7499 & .8264 & 9.317E-11 & $1.221 \mathrm{E}-11$ & 0.1870 & 0.2560 & 0.0683 & 0.3155 \\
\hline Dahurian Birch & .9259 & .8685 & .6467 & .92662 & $1.515 \mathrm{E}-10$ & $2.551 \mathrm{E}-11$ & 3.1645 & 0.3131 & 0.3372 & 0.3409 \\
\hline
\end{tabular}
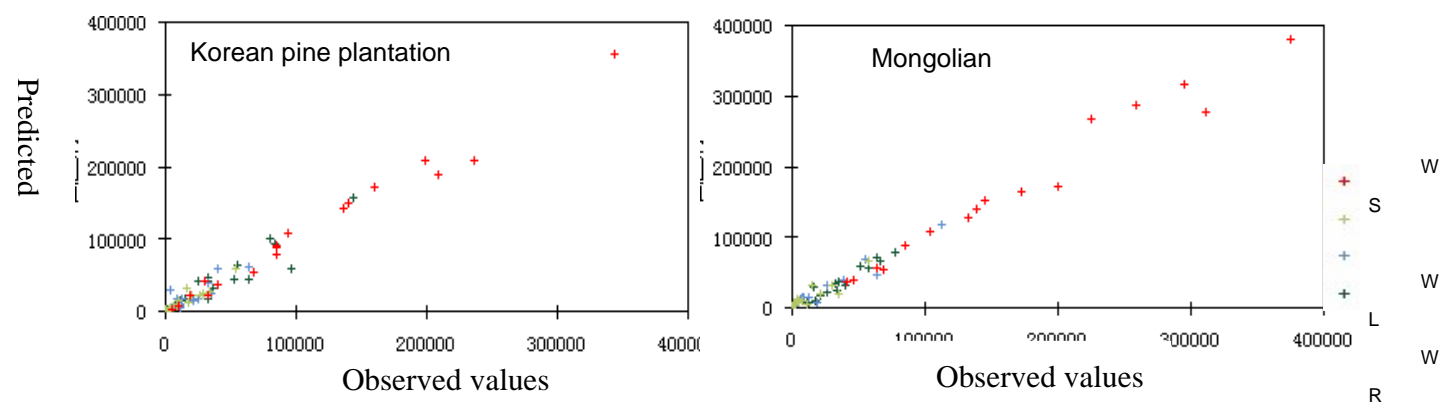

Figure 1 the comparison of predicted valuses-observed values

In Figure 1, Korean pine plantation and Mongolian pine are as delegates to show the predicted The results of the compatible biomass developed above are as follows:

From Table 4, it was seen that the parameter estimation accuracy of compatible biomass equations (average R2 $=0.90$, average $S S E=0.0578$ ) are relative ideal. Mean Bias $(\mathrm{MB})$, and Mean Absolute Bias (MAB) of compatible biomass equations are much less than allowable deviation 0.00001. Moreover the compatible biomass equations could effectively overcome the defect of uncompatible of traditional biomass models, which achieved the purpose of the paper. As was shown in Table 1, traditional biomass models are un-compatible.

From Figure 1, the result can be received that the predicted effect of the compatible biomass model developed in this paper is very reasonable. So the compatible biomass model can be used to predicted forest biomass.

Considering the difficulty of collecting the biomass data and the biomass equation on D explained more than $87.88 \%$ of the biomass variance, this model uses only a single predictor of D and produces a range of prediction values closer to the upper and lower limits of the observed mean, and could be applied to forest biomass estimation in large-scale region.

The effects of fitting the models were better for stem (average $\mathrm{R} 2=0.90$ ) and root (average $\mathrm{R} 2=0.88$ ) and worst for branches (average $\mathrm{R} 2=0.79$ ) and foliage (average $\mathrm{R} 2=0.71$ ), whether traditional biomass models or the compatible biomass developed in the paper.

\section{Conclusion}

The conclusion is that the compatible biomass equations developed in the paper solved the uncompatible of traditional biomass models, which can be widely used in large-scale region of Heilongjiang Province, and the model is reliable to estimate forest biomass, so the research findings can be extrapolated for managing forests related to carbon balance; furthermore the paper provided the constructive method of establishing compatible biomass models for other region or nation. ubmission. 


\section{Acknowledgements}

This research was financially supported by Doctor Startup Fund of Harbin University of Commerce (14rw12), Postdoctoral Startup Fund of Harbin University of Commerce (2017BSH029), the periodical achievement of the general project of the national social science foundation (17BJY119) and Program for Philosophy and Social Science for the Planning of Heilongjiang province (16JYE01)”

\section{References}

[1] Kozak, A. Methods of ensuring biomass additivity of biomass components by regression analysis. Forest Chronology 46(59): 402-404(1974)

[2] Cunia, T. \& Briggs, R.D. Forcing additivity of biomass tables: some empirical results. Canadian Journal of Forest Resources 14: 376-384( 1984).

[3] Parresol, B.R. Assessing tree and stand biomass: a review with examples an critical comparisons. Forest Science 45(4): 573-593 (1999).

[4] Parresol, B.R. Additivity of nonlinear biomass equations. Canadian Journal of Forest Research 31: 865-878 (2001).

[5] Williams, RJ Zerihun, A Montagu, KD. e.t. .Allometry for estimating aboveground tree biomass in tropical and subtropical eucalypt woodlands: towards general predictive equations. Australian Journal of Botany, 53: 670-619 (2005).

[6] Bond-Lamberty, B.; Wang, B. C.; Gower, S.T. Aboveground and belowground biomass and sapwood area allometric equations for six boreal tree species of northern Manitoba. Can. J. For. Res. 32: 1441-1450 (2002).

[7] Shouzheng Tang, Quijian Lang and Haikui Li. ForStat Windows Tutorial. Science Press (2009) 